\title{
A study of dose-response relationships for asbestos associated disease
}

\author{
M M FINKELSTEIN \\ From the Health Studies Service, Ontario Ministry of Labour, Toronto, Ontario M7A IT7, Canada
}

ABSTRACT The risk of an asbestos worker developing small irregular opacities on the chest radiograph is related to cumulative exposure to asbestos dust, latency, and smoking habit. In this study the use of residence-time weighted exposure as a "dose metric" was explored in a cohort of asbestos cement workers. It was found that this parameter, which incorporates both exposure concentration and latency, is useful for modelling the risk of small opacities and might also be useful for modelling the risk of mesothelioma.

After identification of an occupational hazard one of the goals of occupational epidemiology is to quantify the risks by determining the dose-response relations for the toxic agent. In many circumstances little is known about the dose received by target tissues; the data available usually pertain only to exposure to various concentrations of the toxic material in the workplace. The calculation of dose requires additional physiological and chemical information relating to absorption, distribution, biochemical reactions, retention, and clearance.

In asbestos epidemiology the usual measure of exposure is the product of the concentration of asbestos dust in the air (fibres or particles per $\mathrm{ml}$ ) and the duration of exposure to each concentration summed over the entire duration of exposure (years); this measure is the cumulative exposure, expressed symbolically as $E=\Sigma c_{i} t_{i}$ fibres $/ \mathrm{ml} \times$ years (f-y/ml). In a previous study the relations between cumulative exposure to asbestos dust in an asbestos cement factory and the risks of developing various radiographic abnormalities were explored. ${ }^{1}$ The risk of developing small irregular opacities was related both to cumulative exposure and to the time since first exposure (latency) - that is, at any given latency the risk of abnormality increased with cumulative exposure and for any cumulative exposure the prevalence of abnormalities increased with latency. Because of this dependency on both exposure and time, and because radiographic abnormalities are known to develop and progress after exposure has ceased, ${ }^{12}$ it is desirable to use as the dose metric a function of exposure that continues to increase after

Received 30 July 1984

Accepted 17 September 1984 exposure has ended. A family of such dose measures has been discussed by Berry and associates. ${ }^{3}$ The simplest member is an asbestos dose calculated by assuming that a fixed proportion of inspired dust is deposited in the lungs and resides there without clearance; since dust persists in lungs after exposure has ended this measure of dose continues to increase with time. More complex members of this family of dose measures include terms for clearance of fibres and for delayed manifestation of adverse effect. Units for dose are fibres $/ \mathrm{ml} \times$ years-squared $(\mathrm{f} / \mathrm{ml} \times$ $\left.\mathbf{y}^{2}\right)$.

In the present paper the application of these measures of dose to the assessment of risk in an asbestos cement cohort is explored. It was found that the no clearance dose metric provides a useful scale for the measurement of the risk of developing certain radiographic abnormalities and may be useful for assessing the risk of malignant disease as well.

\section{Materials and methods}

\section{STUDY POPULATION}

The workers in the present study were men who had been hired at an asbestos cement factory before 1960 , who had a minimum of nine years' employment with the company and who had been exposed to asbestos for at least one year before 1961. For the radiographic analysis, only the 161 men having a chest film 15 or more years after first exposure were included; 20 men from the earlier cohort ${ }^{1}$ were thus omitted. For the mortality analysis, all 181 men were included. The numbers of men who had follow up films at various intervals after first exposure are given in table 1 , which also shows the distribution of ages at the start of exposure. 
Table 1 Cohort demography

\begin{tabular}{lc}
\hline No of men with radiograph at: & \\
$15-19$ years & 144 \\
$20-24$ years & 122 \\
$25-29$ years & 97 \\
$30-34$ years & 34 \\
Age at start of exposure: & \\
$18-<28$ & $35 \%$ \\
$28-<38$ & $44 \%$ \\
$38-<48$ & $20 \%$ \\
$48-<58$ & $2 \%$ \\
Mean & 31 years \\
Smoking habits: & $72 \%$ \\
Smokers* & $25 \%$ \\
Non-smokers & $2 \%$ \\
Unknown (assumed smokers) & \\
\hline
\end{tabular}

${ }^{*}$ Smokers are defined as men who smoked cigarettes later than 10 years from first exposure.

\section{EXPOSURE AND DOSE ESTIMATION}

Information about the factory and the estimation of individual exposures by extrapolation from personal membrane filter measurements has been published. ${ }^{4}$ Cumulative exposures were calculated for each man by summing annual exposures accumulated during the first 18 years from the start of exposure. Asbestos dosages were calculated by assuming that a fixed proportion of the workplace air concentrations were deposited in the lungs, and each year's accumulation was weighted by the residence time in lung tissue (the formulas used are given in the appendix). Cumulative exposures had been estimated to be accurate to within a factor of 3 to $5^{4}$; since the formulas used to calculate dose weight early exposures most heavily, the dose estimates used here are probably more uncertain than the estimates of cumulative exposure.

\section{SMOKING INFORMATION}

Since the early 1970 s smoking information had been requested by pulmonary function technicians at the time of routine examination. Additional information was obtained by questionnaire and from physicians' records. For the purposes of this study smokers were defined as men who had smoked cigarettes later than 10 years from first exposure. Non-smokers were defined as men who had never smoked or who had stopped before 10 years from first exposure. The smoking status of four men was unknown-they were assumed to have been smokers. For some men the age at the start of smoking and smoking intensity were unknown; for the calculation of pack-years they were assumed to have smoked one package a day from the age of 18 until the time they reported stopping or the time of the radiograph under consideration. Table 1 also gives the distribution of smoking habits.

\section{RADIOGRAPHY}

Chest radiographs had been obtained during routine medical surveillance, in response to an invitation to former employees to return for an examination, or from hospital files. For many men sequential films were available. The films were interpreted, according to the 1971 ILO classification, ${ }^{5}$ by a single NIOSH-certified " $B$ " reader (Dr J J Vingilis) who was unaware of the workers' exposure or smoking histories. The abnormalities selected for study were the small irregular opacities and bilateral pleural thickening, both associated with exposure to asbes- ' tos. For the purposes of regression analysis the categories on the 12 point scale for the small opacities were assumed to be equally spaced and were numbered in integer sequence-beginning with the category $0 / 0$.

\section{DOSE-RESPONSE RELATIONSHIPS}

Dose, in the present paper, is defined as being proportional to the residence-time weighted quantity of dust in the lungs; it is approximated by the dose metric calculated according to the formulas in the appendix. Units are $\mathrm{f} / \mathrm{ml} \times \mathrm{y}^{2}$.

Response is defined as the cumulative risk of developing an abnormality. The dose-response function describes the form of the relation between cumulative risk and the dose metric. Since it was the aim of the analysis to assess the utility of the dose metric in describing risk, the functional form of the dose-response relation was specified beforehand. For the radiographic abnormalities previous analyses had suggested that an S-shaped function (the probit curve) was statistically and biologically plausible as a candidate function. ${ }^{\prime}$ For this analysis the logistic function, which is similar in shape, was selected because of its computational simplicity (appendix).

\section{STATISTICAL METHODS}

Cumulative risk was calculated by life table methods ${ }^{6}$ using dose, rather than time, as the independent variable. When men were withdrawn from the analysis at the end of follow up or at death, they were assigned the dose accumulated to that year.

The influence of confounding factors was assessed $\sigma$ by the Cox regression analysis ${ }^{7}$ and by multiple $N$ linear regression. The logistic dose-response function was fitted to the cumulative risk by weighted linear regression. ${ }^{8}$

\section{Results}

\section{ASSESSMENT OF RISK FACTORS}

In the previous analysis smokers were at higher risk of developing radiographic abnormalities than non- 
Dose-response relationships for asbestos associated disease

Table 2 Coefficients from Cox regression analysis of radiographic abnormalities

\begin{tabular}{|c|c|c|c|c|c|c|c|c|}
\hline \multirow[t]{2}{*}{ Abnormality } & \multicolumn{6}{|l|}{ Variable } & \multirow[t]{2}{*}{ Model $\chi_{3}^{2}$} & \\
\hline & $\begin{array}{l}\text { Exposure } \\
\beta^{*}\end{array}$ & $P$ & $\underset{\beta}{\text { Smoking }}$ & $P$ & $\begin{array}{l}\text { Age } \\
\beta\end{array}$ & $P$ & & \\
\hline $\begin{array}{l}\text { Small opacities } \geqslant 1 / 0 \\
\text { Small opacities } \geqslant 1 / 1 \\
\text { Small opacities } \geqslant 1 / 2 \\
\text { Pleural thickening } \geqslant A \\
\text { Pleural thickening } \geqslant B\end{array}$ & $\begin{array}{l}6.3 \times 10^{-3} \\
6.8 \times 10^{-3} \\
1.7 \times 10^{-2} \\
1.2 \times 10^{-3} \\
8.5 \times 10^{-4}\end{array}$ & $\begin{array}{c}<0.001 \\
<0.002 \\
<0.001 \\
0.32 \\
0.39\end{array}$ & $\begin{array}{l}0 \cdot 60 \\
0 \cdot 93 \\
1 \cdot 4 \\
1 \cdot 2 \\
1 \cdot 1\end{array}$ & $\begin{array}{l}0.047 \\
0 \cdot 014 \\
0 \cdot 10 \\
0 \cdot 007 \\
0.095\end{array}$ & $\begin{array}{c}0.041 \\
0.051 \\
-0.02 \\
0.042 \\
0.050\end{array}$ & $\begin{array}{l}0 \cdot 039 \\
0 \cdot 011 \\
0 \cdot 67 \\
0 \cdot 046 \\
0 \cdot 055\end{array}$ & $\begin{array}{r}20 \cdot 8 \\
21 \cdot 8 \\
14 \cdot 7 \\
12 \cdot 7 \\
7 \cdot 2\end{array}$ & $\begin{array}{l}\mathrm{p}<0.001 \\
\mathrm{p}<0.001 \\
\mathrm{p}<0.01 \\
\mathrm{p}<0.01 \\
\mathrm{p}=0.07\end{array}$ \\
\hline
\end{tabular}

${ }^{*} \beta$ is regression coefficient. Units for exposure are $\mathrm{f}-\mathrm{y} / \mathrm{ml}$.

$\mathrm{P}$ is one-sided probability that a coefficient as large, or larger than, $\beta$ would occur by chance.

smokers.' In that calculation age had not been controlled for; to assess the simultaneous influence of cumulative exposure, age, and smoking on risk a Cox regression analysis was used to model the time to development of abnormalities among the 147 men who had normal films at 18 years latency. Variables in the model included 18 years cumulative exposure, age at 18 years latency, smoking habit (binary yes/no variable), and a smoking-exposure interaction term; the results are presented in table 2 .

For the small opacities, cumulative exposure was a significant contributor to risk, as were both smoking and age. The smoking-exposure interaction term was not significant for any category of opacities. Relative risk in this model is approximated by $\mathrm{e}^{\beta}$ and point estimates of relative risk for smokers were 1.8 for opacities $\geqslant 1 / 0,2 \cdot 5$ for opacities $\geqslant 1 / 1$, and $4 \cdot 1$ for opacities $\geqslant 1 / 2$.

The results were qualitatively different for bilateral pleural thickening, for which cumulative exposure was unrelated to the risk of developing an abnormality. This finding is similar to that of the earlier analysis' in which a trend with exposure was found only when men lost to follow up (who were assumed to have normal films) were included in the calculations. Smoking habit and age each contributed to the model for pleural thickening but the amount of variability accounted for was small.

\section{MEAN RADIOGRAPHIC SCORE VERSUS DOSE}

For the dose metric to be a useful improvement on cumulative exposure in the evaluation of risk, predictions made with it should be independent of latency-that is, if each member of one group of workers accumulates a dose, D, by 20 years after first exposure and another group reaches the same dose at 35 years latency, then the prevalence of abnormalities should be the same in both groups at the time $D$ is reached.

An evaluation of latency dependence of the risk assessment was obtained when multiple linear regression (MLR) was used to model the relation between radiographic score and the dose metric. For each of the time intervals $15-19,20-24,25-29$, and
30-34 years from first exposure, regression models were constructed using the radiographic codes from each film obtained in that interval as the response variables and dose, age, and smoking as predictor variables. Residuals were examined to assess the validity of the models. There were so few abnormal films before 20 years that the residuals were distributed in a highly skewed fashion and the MLR model was judged to be inappropriate. For the other time intervals the model assumptions of normality and homoscedasticity appeared to be reasonably well met and the model was judged to be valid. Thus although the individual radiographic scores are described on the integer scale, the regression line appears to be a valid descriptor of the average score at any dose.

For the small opacities dose contributed significantly to the prediction of mean radiographic score in all time intervals. Both age and smoking were significant predictors when smoking entered the model as a binary variable, but when smoking was expressed as the quantitative pack-years variable, age was no longer statistically significant, suggesting that in the binary models age assumed importance because of the correlation between age and pack-years $(r=0 \cdot 49)$. As with the Cox models, a dose-smoking interaction term was not significant. The mean radiographic score was increased one category by a 50 pack-year smoking history ( $95 \%$ confidence limits: $30-70$ pack-years) and by an asbestos dose of about $2000 \mathrm{f} / \mathrm{ml} \times$ years $^{2}$ (recall the large uncertainty in dose estimation). The standard error about the mean score was approximately 1.3 categories so that there was wide variation in response; as measured by the multiple $-R^{2}$ these models accounted for only about one third of the variability in the radiographic scores. This is a common finding in occupational epidemiology."

To test whether the dose metric produced predictions that were independent of latency, indicator variables for the time of examination were introduced into the regression models and F-tests were performed to examine whether the expanded models improved the fit to the data. The null hypothesis 
Table 3 Coefficients from cumulative risk function*

\begin{tabular}{|c|c|c|c|c|}
\hline Abnormality & $\begin{array}{l}\text { Smokers } \\
a\end{array}$ & $b$ & $\begin{array}{l}\text { Non-smokers } \\
a\end{array}$ & $b$ \\
\hline $\begin{array}{l}\text { Small opacities } \geqslant 0 / 1 \\
\text { Small opacities } \geqslant 1 / 0 \\
\text { Small opacities } \geqslant 1 / 1 \\
\text { Small opacities } \geqslant 1 / 2\end{array}$ & $\begin{array}{l}-4 \cdot 88 \\
-4 \cdot 94 \\
-4 \cdot 94 \\
-5 \cdot 84\end{array}$ & $\begin{array}{l}0 \cdot 0021 \\
0 \cdot 0019 \\
0 \cdot 0016 \\
0 \cdot 0011\end{array}$ & $\begin{array}{l}-4 \cdot 0 \\
-4 \cdot 7 \\
-4 \cdot 5\end{array}$ & $\begin{array}{l}0 \cdot 0012 \\
0.0013 \\
0 \cdot 0010\end{array}$ \\
\hline
\end{tabular}

It must be recalled that the estimates of dose used to calculate these coefficients have a large margin of uncertainty.

of time-independence was not rejected, and it was concluded that the dose metric could be used to predict mean radiographic scores in this population over the time interval $20-34$ years from first exposure.

An attempt was made to model scores for bilateral pleural thickening but this ended in failure; there was no association between pleural thickening and the dose metric in this cohort.

\section{DOSE-RESPONSE RELATIONSHIPS}

Cumulative risks were calculated separately for smokers and non-smokers by life table methods. To assess the latency dependence of cumulative risk, "survival curves" giving cumulative risk versus dose were calculated for the end of $19,24,29$, and 34 years of follow up. A graphical comparison indicated that for all practical purposes the doseresponse function was independent of latency over the period 20-34 years from first exposure.

The logistic risk function was fitted to the estimates of cumulative risk by weighted linear regres-

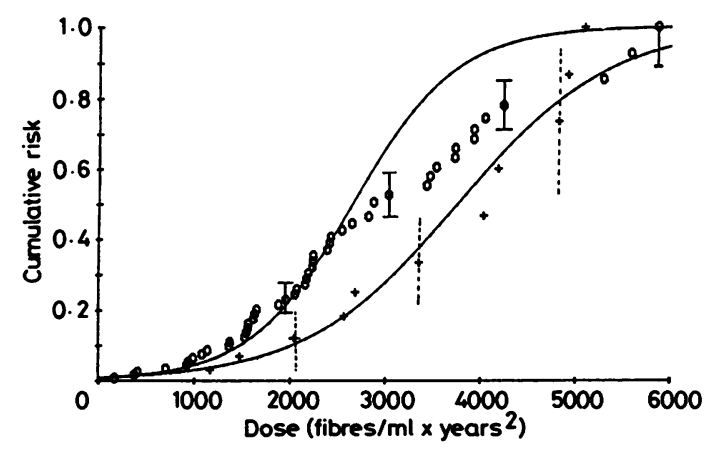

Fig 1 Cumulative risk of developing small irregular opacities $\geqslant 1 / 0$ versus dose for smokers (circles) and non-smokers (crosses). Error bars are estimates of standard error of cumulative risk and curves are logistic functions futted to data.
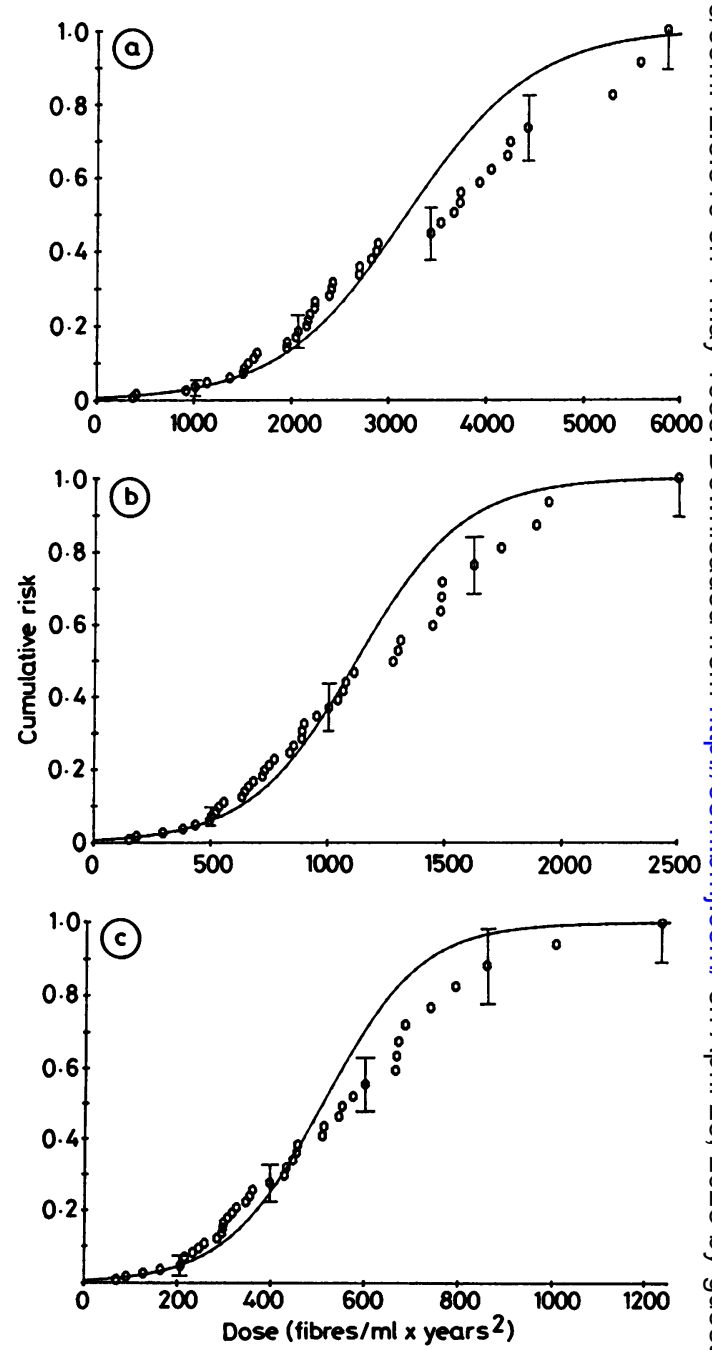

Fig 2 Cumulative risk of small irregular opacities $\geqslant 1 / 1$ versus dose. Dose metric assumes no clearance in $(A)$, and clearance half times of five years in $(B)$ and two years in $(C)$. Curves show logistic functions futed to data. 


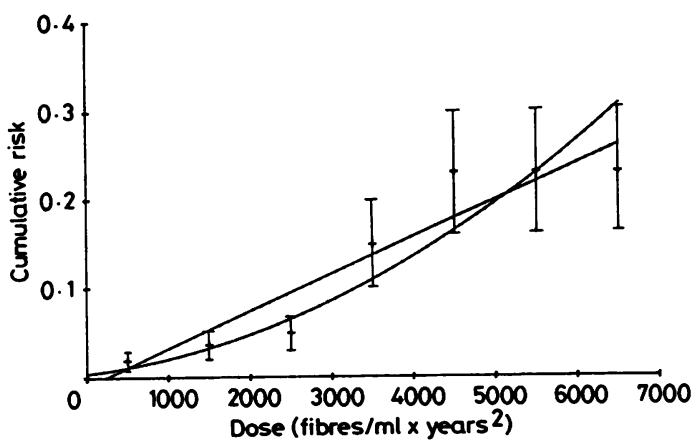

Fig 3 Cumulative risk of death from mesothelioma versus dose. Error bars give estimates of standard error of risk and curves are linear and quadratic functions futted to data.

sion and, as judged by the F-statistic for regression, the logistic model provided an adequate fit to the data. The coefficients of the logistic equations are given in table 3 and the curves for small opacities $\geqslant 1 / 0$ in fig 1 .

Two additional dose metrics, incorporating clearance half times of two years and five years, were computed and the corresponding dose-response functions were calculated. These are illustrated in fig 2 for the small opacities $\geqslant 1 / 1$. There was little difference among these measures of dose with respect to adequacy of fit of the logistic function, and the simplest one, the no clearance model, is thus most useful for risk estimation.

\section{MORTALITY}

To investigate whether the dose metric might be a useful descriptor of risk for a mortality endpoint, the 12 deaths from mesothelioma among the cohort were examined. Cumulative risk was calculated by the life table method, and because the incidence of mesothelioma is believed to have a power function relation with time ${ }^{10}$ and incidence is related to the derivative of cumulative risk (see appendix), an attempt was made to fit a power function to the relation between cumulative risk and dose. As illustrated in fig 3 , both linear and quadratic functions gave equally good fits to the data.

\section{Discussion}

The traditional method of measuring exposure to hazardous dusts in epidemiological studies of the pneumoconioses is by the calculation of the cumulative exposure. This measure, which may be interpreted as implying immediate clearance of dust from the lungs, suffers from the shortcoming that its value remains fixed once exposure ends, by contrast with biological endpoints that may change as time passes. An alternative approach was first proposed by Jahr," who suggested that each increment of dust exposure should be weighted by the time since it was accumulated. Berry et al proposed more advanced models that permitted clearance of dust and the expression of a lag time between dust accumulation and the appearance of an adverse effect. ${ }^{3}$ In the present study the application of these measures of dose to the assessment of risk in a cohort of asbestos cement workers was explored. It was found that the simple no clearance model could be used to predict both the cumulative risk of development of small irregular opacities and the average radiographic code in this group of workers. These descriptions were found to have the desirable property of being independent of the time of evaluation up to 35 years latency. It is thus possible to describe risk in terms of one parameter, dose, rather than the two, cumulative exposure and latency, required with the traditional measure of exposure. Whether these descriptions remain valid beyond 35 years of latency will require future assessment.

The various measures of dose examined should be looked on as mathematical models for the description of risk rather than as descriptors of physiological processes. The inability to select a model incorporating a clearance term as being preferable to one which ignores clearance thus means that the simpler mathematical model is suitable for describing risk but has no implications for the actual physical clearance of fibres from the lungs. Clearance is known to occur, but the epidemiological method is probably inappropriate for the investigation of the dynamics of the clearance processes.

In the present study the development of small irregular opacities could be successfully modelled by this approach, but there was no association between dose and the risk of bilateral pleural thickening. A previous analysis had found that when the cohort was expanded to include men lost to follow up (who were assumed to remain free of pleural thickening) then an association between exposure and risk did appear. ' Liddell et al were unable to show a relation between exposure and pleural thickening among Quebec chrysotile miners after many years of service. ${ }^{12}$ Therefore, it is not clear whether there is a quantitative relation between exposure to asbestos and the risk of pleural thickening which is so weak that it is obscured by uncertainties in the estimation of exposure and the interpretation of films or whether the risk of developing pleural thickening is determined primarily by factors other than the quantity of dust inhaled. In this analysis age and smoking habit were found to have weak discriminatory powers for pleural thickening but the 
amount of variability accounted for was low.

It is a well known phenomenon that individuals exposed in apparently similar circumstances to hazards such as noise or mineral dusts may have dissimilar biological responses. Some men exposed to relatively low concentrations of dust may develop fibrosis or tumours whereas others exposed to much greater quantities of dust may never show any adverse effect. In this analysis, when modelling the relation between radiographic code and dose, and taking account of smoking and age, it was possible to account for only about one third of the variability in response. Similarly, Liddell et al were able to account for only about one third of the variability in radiographic progression among Quebec miners, and the explanatory variable accounting for most of the total variation was usually not a measure of exposure. ${ }^{9}$ Few factors that modify the risk (one of them is cigarette smoking) have been identified and it is thus possible, in general, to make predictions only for groups rather than for individuals.

The influence of smoking in this cohort was examined by assessing its contribution to the radiographic code through multiple linear regression analysis, and to the risk of developing an abnormality in the Cox survival analysis. In both analyses dust exposure and smoking each contributed to risk and a dose-smoking interaction term made no significant contribution to the fit of the models-that is, the relative risk for smokers, compared with nonsmokers, at the same dose, was independent of their asbestos doses.

Mortality from malignant mesothelioma was selected as the endpoint for examination of whether the dose metric might be a useful predictor of the risk of death from malignant disease. Mesothelioma is suitable for this purpose because the mortality rates are believed to be independent of age and smoking habit but related to exposure and latency. Peto et al investigated mortality rates among several cohorts exposed to asbestos and found that incidence rates could be described by a power function relation with time, having an exponent somewhere between 2 and $4 .^{10}$ As shown in the appendix, incidence is proportional to both cumulative exposure and the derivative of cumulative risk with respect to dose. Observations in this cohort are compatible with a quadratic dose-response relation, which would lead to incidence rates that vary approximately with $\mathrm{T}^{3}$ while exposure is continuing and with $T$ after exposure has ended (fig 3 ). These observations imply that this dose metric might be useful for modelling mesothelioma risk but because of the small size of this population no definitive statement can be made and the matter will require verification in other cohorts.

\section{Appendix}

FORMULAS FOR THE CALCULATION OF DOSE ${ }^{3}$ : For the no clearance model

and in general

$$
D(t)=\int_{0}^{t}(t-u) c(u) \mathrm{d} u
$$

$$
D(t)=\frac{1}{\lambda} \int_{0}^{t} c(u)\left[1-\mathrm{e}^{-\lambda(t-u)}\right] \mathrm{d} u
$$

where $c(u)$ is the concentration at time $u$

$$
\text { and } \lambda=\frac{\ln 2}{T}
$$

where $T$ is the clearance half time.

LOGISTIC DOSE-RESPONSE EQUATION:

$$
C R=\frac{\mathrm{e}^{a+b D}}{1+\mathrm{e}^{a+b D}}
$$

where CR is the cumulative risk, $a$ and $b$ are parameters to be estimated, and $D$ is the dose.

RELATION BETWEEN INCIDENCE AND CUMULATIVE RISK:

$$
\begin{aligned}
I(t) & =\frac{\mathrm{d}}{\mathrm{d} t}(\mathrm{CR}) \\
& =\frac{\mathrm{d}(\mathrm{CR})}{\mathrm{d} D} \times \frac{\mathrm{d} D}{\mathrm{~d} t}
\end{aligned}
$$

Using (i) above,

$$
\frac{\mathrm{d} D}{\mathrm{~d} t}=\int_{0}^{t} c(u) \mathrm{d} u
$$

Thus

$$
I(t)=\frac{\mathrm{d}(\mathrm{CR})}{\mathrm{d} D} \times \int_{0}^{t} c(u) \mathrm{d} u .
$$

If, for mesothelioma, $\mathrm{CR}=K D^{2}$.

Then

$$
I(t)=2 k D \int_{0}^{t} c(u) \mathrm{d} u
$$

which varies approximately as $t^{3}$ when exposure is continuing and as $t$ after exposure has ended.

\section{References}

' Finkelstein MM, Vingilis JJ. Radiographic abnormalities among asbestos-cement workers; an exposure response study. Am Rev Respir Dis 1984;129: 17-22.

${ }^{2}$ Viallat JR, Boutin C, Pietri JF, Fondari J. Late progression of radiographic changes in Canari chrysotile mine and mill $O$ exworkers. Arch Environ Health 1983;38:54-8.

${ }^{3}$ Berry G, Gilson JC, Holmes S, Lewinsohn H, Roach S. Asbestosis: a study of dose-response relationships in an asbestos? textile factory. Br J Ind Med 1979;36:98-112.

${ }^{4}$ Finkelstein MM. Asbestosis in long-term employees of an 0 Ontario asbestos-cement factory. Am Rev Respir Dis 1982;125:496-501

${ }^{s}$ International Labour Office. ILO U/C international classification 
of radiographs of pneumoconioses 1971. (Occupational safety and health series 22 (rev).) Geneva: International Labour Office, 1972.

- Thomas DG, Breslow N, Gart JJ. Trend and homogeneity analyses of proportions and life table data. Comp Biomed Res 1977; 10:373-81.

' Harrell F. The PHGLM procedure. (SAS supplemental library user's guide.) Cary, North Carolina: SAS Institute Inc, 1980.

${ }^{*}$ Chatterjee S, Price B. Regression analysis by example. New York: John Wiley and Sons, 1977.

${ }^{4}$ Liddell FDK, Eyssen G, Thomas D, McDonald JC. Radiological changes over 20 years in relation to chrysotile exposure in Quebec. In: Walton WH, ed, Inhaled particles $I V$. Oxford: Pergamon Press, 1977:799-813.

10 Peto J, Seidman H, Selikoff IJ. Mesothelioma mortality in asbestos workers: implications for models of carcinogenesis and risk assessment. Br J Cancer 1982;45: 124-35.

" Jahr J. Dose-response basis for setting a quartz threshold limit value. Arch Environ Health 1974;29:338-40.

12 Liddell FDK, Gibbs GW, McDonald JC. Radiological changes and fibre exposure in chrysotile workers aged $60-69$ years at Thetford Mines. Ann Occup Hyg 1982;26:889-98. 